$\Rightarrow$ OSTEOARTHRITIS

\section{IL-36 inhibition to treat OA}

Interfering with transforming growth factor- $\beta$ (TGF $\beta$ ) signalling by targeting IL-36 family cytokines could have disease-modifying effects in osteoarthritis (OA), according to new research in mouse models of OA and in tissue from patients with the disease. Li et al. suggest that IL-36 receptor antagonist (IL-36RN in humans; IL-36Ra in mice) might be a future therapy for OA.

Previous studies have implicated altered TGF $\beta$ signalling in the development and progression of OA, but global inhibition of TGF $\beta$ activity could have negative multi-organ effects, suggesting the need for alternative approaches. The present study demonstrates the feasibility of attenuating OA by targeting a TGF $\beta$ type 2 receptor (TGFBR2)IL-36 signalling axis relevant to joint development and homeostasis.

Li et al. demonstrated that Tgfbr2 $2^{-/-}$mice spontaneously developed OA and also had more severe post-traumatic OA (induced by destabilization of the medial meniscus (DMM)) than control mice. Genetic ablation of $T g f b r 2$ was accompanied by increased expression of IL-36 $\alpha$ and IL-36 receptor (IL-36R) and decreased expression of IL-36Ra; similar expression patterns of these cytokines and receptors were observed in wild-type mice treated with a TGF $\beta$ inhibitor (SB-505124), in ageing mice with naturally occurring $\mathrm{OA}$ and in wild-type mice with DMM-induced post-traumatic OA.

Consistent with these findings, blocking TGFBR2 signalling in primary cultures of human articular chondrocytes with SB-505124 led to dose-dependent increases in IL-36 $\alpha$, IL-36R and matrix metalloproteinase 13 (MMP13) and a decrease in IL-36RN. Furthermore, examination of articular cartilage tissue from human donors (with or without OA-like degenerative changes) revealed a pattern of gradually decreasing TGFBR2 expression and gradually increasing IL-36 $\alpha$, IL-36R and MMP13 expression that correlated with increasing severity of cartilage degeneration.

Demonstrating the therapeutic potential of targeting the TGFBR2IL-36 axis, intra-articular injection of IL-36Ra attenuated OA progression in $T g f b r 2^{-/-}$mice as well as in wild-type mice with DMM-induced OA; by contrast, injection of IL-36a exacerbated OA in both models. In human articular chondrocytes from donors with end-stage OA, treatment with IL-36RN led to a dose-dependent decrease in MMP13 expression in vitro.

Together, the results of the study suggest that targeting IL-36 signalling could attenuate the OA process.

Sarah Onuora

ORIGINAL ARTICLE Li, T. et al. TGF- $\beta$ type 2 receptor-mediated modulation of the IL-36 family can be therapeutically targeted in osteoarthritis. Sci. Transl. Med. 11, eaan2585 (2019)

\title{
Two targets are better than one
}

Although several therapeutic options exist for treating rheumatoid arthritis (RA), not even the current gold standard therapies can reliably induce long-term, drug-free remission. Owing to the complex pathogenic processes involved in RA, combination therapies are being developed to target multiple pathways or cytokines at once.

In a new study, one such combination therapy has shown promise in inducing long-term remission in mice with collagen-induced arthritis (CIA). "Given the high costs of biologic therapies and potential for adverse events, we became interested in the therapeutic potential of small molecular weight modulators of TNF receptor signalling," explains corresponding author Richard Williams. "We subsequently started to investigate inhibitors of cellular inhibitor of apoptosis proteins (cIAPs), which have a fundamental role in NF- $\kappa$ B pathway activation by TNF."

The cIAP1 and cIAP2 antagonist GT13072 belongs to a class of inhibitors known as SMAC mimetics that are currently in phase I testing for the treatment of cancer. The researchers used GT13072 to treat mice with CIA and observed a large and rapid decrease in disease severity. Intriguingly, mice treated with GT13072 had reduced numbers of $\mathrm{IL}-17^{+} \mathrm{T}$ cells compared with mice treated with a structurally related inactive compound. "A more detailed look into the mechanism of action of GT13072 revealed that it decreased NFATc1 expression in human T cells, which is a known regulator of IL-17A expression," says first author Joanna Kawalkowska.

As inhibition of TNF can cause an increase in $\mathrm{IL}-17^{+}$cells in patients with $\mathrm{RA}$, the researchers decided to counter this effect by combining the TNF inhibitor etanercept with GT13072 to treat mice with CIA. Combination therapy had an additive effect on reducing disease severity in these mice and also prevented relapse for longer than treatment with either therapy alone.

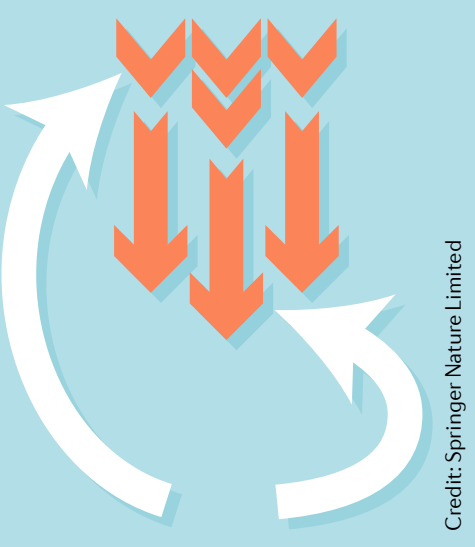

"Perhaps the most important finding from this study was the long-term therapeutic effect of combination therapy, accompanied by an expansion of regulatory T cells," concludes Williams. "Our objective is to translate these findings into the clinic and to initiate experimental medicine trials in patients with inflammatory diseases."

Joanna Collison

ORIGINAL ARTICLE Kawalkowska, J. Z. et al.

cIAP1/2 inhibition synergizes with TNF inhibition

in autoimmunity by down-regulating IL-17A and inducing $\mathrm{T}_{\text {regs }}$. Sci. Adv. 5, eaaw5422 (2019) 